\title{
Evidence Disclosure and Verifiability
}

\author{
Jesse Bull and Joel Watson*
}

June 2000, Revised September 2002

\begin{abstract}
We explore the notion of "verifiability" by analyzing a simple model of evidence production in contractual relationships with complete information. We characterize implementability in terms of the existence and form of "hard evidence." We provide results on maximal and minimal evidence production that are, respectively, necessary and sufficient for implementation. We briefly discuss the relevance of our results to actual legal institutions. JEL Classification: Cro, D74, K10.
\end{abstract}

The notion of "verifiability" — that a court or other external enforcer can observe a given aspect of a contractual relationship - is at the heart of the contract-theory literature. Economic models of contract generally start with a specification of things considered verifiable and then assume that court-enforced contracts can condition transfers arbitrarily on, and only on, these things. In reality, however, court action is a function not of what can be observed by the court but what evidence is actually presented to the court by the contracting parties. Thus, verifiability critically depends on the parties' incentives to submit evidence.

In this paper, we design a simple model to study how evidentiary incentives influence implementation. We examine contractual settings with complete information and externally enforced monetary transfers. In our model, the players first agree to a contract. Then they engage in productive interaction, which results in the "state" of the relationship. After the state is realized, the players can voluntarily submit evidence to the court, who compels monetary transfers contingent on evidence, as directed by the players' contract. Evidence is represented as physical documents, the existence of which depends on the state. We study the agents' incentives to disclose

\footnotetext{
${ }^{*}$ University of California, San Diego. Internet: http://weber.ucsd.edu/ jwatson/. The authors are especially grateful to Jennifer Reinganum (the editor in charge of this paper) and two anonymous referees, whose insightful comments directed a revision of this paper. The authors also thank the following people for their comments: Sandeep Baliga, Vince Crawford, Steven Goldman, Christian Haefke, Ehud Kalai, Jean-Jacques Laffont, Mark Machina, Joel Sobel, Lars Stole, Chris Woodruff, and seminar participants at Columbia University, Northwestern University, UC San Diego, University of Rochester, and Washington University.
} 
documents and we characterize how the form of available evidence imposes limits on the implementation of state-contingent transfers.

Our analysis characterizes implementability in terms of the existence and form of "hard evidence," where this term refers to documents that exists in some states but not in others. We show that implementing different transfers across states requires hard evidence that distinguishes between the states. Further, we show how implementation is sensitive to who possesses evidence and whether the evidence is positive or negative in nature.

Regarding evidentiary forms, consider a setting in which there are two players and two possible states, $a$ and $b$. Suppose that player 1 possesses document $d$ in state $a$, but that in state $b$ no documents exist. We say that disclosure of $d$ is positive evidence of $a$, while nondisclosure of $d$ is negative evidence of $b$. Imagine that the players wish to induce a transfer $t$ from player 1 to player 2 in state $a$ and no transfer in state $b$. To implement this transfer schedule, the players must write a contract instructing the court to compel transfer $t$ if and only if document $d$ is disclosed. However, to make this work, player 1 must have the incentive to disclose $d$ in state $a$, which implies that the transfer schedule is implementable if and only if $t \leq 0$. Our main results generalize this idea; a player gets a higher transfer in state $a$ than he does in state $b$ if and only if either he has positive evidence of $a$ or the other players have positive evidence of $b$, or both. Overall, our analysis associates verifiability with a partition of the state space that the distribution of documents defines. The nature of evidence imposes additional restrictions. ${ }^{1}$

We also provide results on the amounts of evidence that are necessary and sufficient for implementation. Our "full disclosure result" justifies restricting attention to behavioral rules in which, in every state, the players submit all of the documents in their possession. Full disclosure is not necessary for implementation, however; another of our theorems describes minimal sets of documents that are required for implementation.

Because we study settings of complete information, our model is one of "Nash implementation" (Maskin 1999). It is not a standard mechanism-design model, however, because it specifies inalienable evidentiary decisions and state-contingent evidence sets. That is, in mechanism-design language, the model has fixed message spaces and state-dependent restrictions on what the players can say. This type of setting was previously analyzed by Green and Laffont (1986). ${ }^{2}$ There some interpretive and minor technical differences between our model and that of Green and Laffont; these are discussed in the companion paper, Bull and Watson (2002), which stud-

\footnotetext{
${ }^{1}$ This leads to a smaller implementable set than is sometimes assumed in the literature, which typically represents verifiability simply as a partition of the state space. Examples of this approach include Holmström (1982), Legros and Matthews (1993), and Bernheim and Whinston (1999).

${ }^{2}$ Our notion of documents existing in different contingencies is also like the reporting constraints studied by Milgrom and Roberts (1986), Okuno-Fujiwara, Postlewaite and Suzumura (1990), Shin (1994), Lipman and Seppi (1995), and Seidmann and Winter (1997); and it resembles the "sending a message cannot be verified" setting studied by Hart and Moore (1988).
} 
ies the relation between models of hard evidence and "abstract-declaration" models. As we discuss in the next section, our conclusions are not related to those of Green and Laffont. Rather, our results follow from the special nature of "public decisions" in our model, whereby the court only compels transfers. Further, justified by the consideration of renegotiation (as in Maskin and Moore 1999), we assume that the transfers are balanced, meaning that they sum to zero. In this contracting environment, evidence production with court enforcement is a zero-sum game. We discuss why this additional structure, relative to general mechanism-design environments, is somewhat realistic. As we focus on the opportunities that contracting parties have to present documented evidence, our modeling approach differs from other models of court decision making and evidence production. ${ }^{3}$

The first part of this paper presents the analysis for the case of two players. Section 1 describes the basic model and Section 2 contains the characterization results. In Section 3, we use the model to discuss some features of actual legal institutions. In particular, we note that U.S. law differentiates between positive and negative evidence in a way that is roughly consistent with our model. We also use the model to describe the effect of having a strong "enforced discovery" system, which reduces the problem of negative evidence, but we argue that such a system is typically infeasible.

In Section 4, we extend our results to settings with more than two players. We show that all of our findings, with the exception of one, extend to the $n$-player environment under an additional assumption about side deals. Specifically, we utilize a version of Bernheim, Peleg, and Whinston's (1987) coalition-proofness concept, requiring that the players' initial contract be impervious to side-contracting by coalitions. Under the side-contracting constraint, hard evidence is critical for implementation and "message game phenomena" cannot arise.

Appendix A contains proofs not found elsewhere in the text. In Appendix B, we present a simple model of productive interaction along the lines of Holmström (1982) and Legros and Matthews (1993). We characterize the productive action profiles that can be induced, given the set of implementable state-contingent transfers.

\footnotetext{
${ }^{3}$ The influence-cost literature studies models in which the probability of a litigant winning at trial is a function of the litigants' expenditures on evidence. See, for example, Tullock (1980), and Cooter and Rubinfeld (1989). There are also studies of the court as a Bayesian decision maker who receives signals of the defendant's type. The signals are influenced by effort or expenditure in evidence production. See for, example, Rubinfeld and Sappington (1987). Modeling the court as a Bayesian decision maker may not be appropriate in all settings, however (Daughety and Reinganum 2000a). The strategic search literature models each party's evidence production as a costly random draw of evidence from a distribution of evidence. The most favorable evidence drawn is assumed to be presented in court. This allows for incomplete information between the parties. Examples of this approach include Daughety and Reinganum (2000b), and Froeb and Kobayashi (2000). An advantage of this costly sampling approach is that it allows for the consideration of evidence costs. However, it does not address multiple dimensions of evidence production, or the cost of individual pieces of evidence.
} 


\section{The Basic Model}

This section describes our basic two-player model of evidence and external enforcement. Following the development of the model are brief interpretive and technical notes.

\section{Timing and Definitions}

We consider a contractual relationship between 2 players (also called agents), who interact over four periods of time. In the first period, the players form an externally enforced contract, which specifies monetary transfers to be compelled by the court as a function of evidence that the players will later present. Technically, the players' contract defines a function $m: \mathcal{D} \rightarrow \mathbf{R}_{0}^{2}$, where $\mathcal{D}$ is the space of possible evidence (discussed below) and

$$
\mathbf{R}_{0}^{2} \equiv\left\{\left(x_{1}, x_{2}\right) \in \mathbf{R}^{2} \mid x_{1}+x_{2}=0\right\}
$$

describes the set of balanced transfers. We write $m=\left(m_{1}, m_{2}\right)$, so that, for $i=1,2$, $m_{i}$ gives the transfer to player $i$.

In the second period, all productive interaction occurs; this may include, among other things, investments and trading decisions that the players make, as well as any random draws. The productive interaction determines an event $a$ that we call the state of the relationship. The state is commonly observed by the players. We let $A$ denote the set of possible states and we assume that $A$ is finite. Our purposes do not require modeling the details of productive interaction, because we shall primarily be concerned with how externally enforced transfers can be made contingent on the state. ${ }^{4}$

External contract enforcement occurs in the last two periods. Specifically, in period 3 the players simultaneously and independently present evidence to the court. Then, in period 4, the court compels the monetary transfer specified by $m(E)$, where $E$ denotes the evidence that the players have submitted. In the end, player $i$ 's payoff is given by $u_{i}(a)+m_{i}(E)$, for some function $u_{i}: A \rightarrow \mathbf{R}$. Note that payoffs are additive in the externally enforced transfer. In most of our analysis (except in Appendix B), we can ignore the $u$ component of payoffs.

We model evidence as a set of documents, whose existence depends on the state. We denote by $D_{i}(a)$ the set of documents that can be presented by player $i$ in state $a$. Since not all documents may be available in all states, $D_{i}(a) \neq D_{i}(b)$ is generally, but not necessarily, the case. Let $D_{i} \equiv \bigcup_{a \in A} D_{i}(a)$ denote the set of documents available to player $i$ over all states. We assume $D_{i}$ is finite. We also assume $D_{1}$ and $D_{2}$ are disjoint sets and we let $D \equiv D_{1} \cup D_{2}$ and $D(a) \equiv D_{1}(a) \cup D_{2}(a)$ for each $a$. Assuming $D_{1}$ and $D_{2}$ are disjoint is without loss of generality; we make this assumption for convenience, so each document can be unambiguously associated with one player. For any set of documents $E \subset D$, we write $E_{i}$ as those documents disclosed by

\footnotetext{
${ }^{4}$ See Appendix B for a simple model of productive interaction.
} 
player $i$. Each player can freely disclose any subset of his existing documents. Thus, the feasible evidence sets are given by

$$
\mathcal{D} \equiv\{E \mid E \subset D(a) \text { for some } a \in A\}
$$

Note that each player always has the option of disclosing no documents.

The court-enforced function $m$, along with the realized state $a$, implies an evidencedisclosure game that is played in period 3. In this game, player $i$ 's strategy space is given by $\left\{E_{i} \mid E_{i} \subset D_{i}(a)\right\}$ and payoffs are defined by $m$. We apply the term disclosure rule to any function $\beta: A \rightarrow \mathcal{D}$ that satisfies $\beta(a) \subset D(a)$ for each $a \in A$. Such a function describes how the players behave in the evidence-disclosure game in each state. $^{5}$ That is, in state $a$ the players disclose document set $\beta(a)$. Let $\beta_{i}(a)$ denote the documents presented by player $i$ in state $a .^{6}$

Definition 1: Given $m$, we call $\beta$ an equilibrium disclosure rule if $\beta$ specifies a Nash equilibrium for each a; that is,

$$
m_{i}(\beta(a)) \geq m_{i}\left(E_{i} \cup \beta_{-i}(a)\right)
$$

for each $i \in N, E_{i} \subset D_{i}(a)$, and $a \in A$.

We call any $g: A \rightarrow \mathbf{R}_{0}^{2}$ a transfer function; it describes the transfer in period 4 that occurs in each state. An externally enforced contract $m$ and a disclosure rule $\beta$ imply the transfer function $g$ that is defined by $g(a)=m(\beta(a))$ for every $a \in A$.

Definition 2: A transfer function $g$ is called implemented by externally enforced contract $\boldsymbol{m}$ and disclosure rule $\boldsymbol{\beta}$ if (i) $\beta$ is an equilibrium relative to $m$ and (ii) $g(a)=m(\beta(a))$ for every $a \in A$. Also, $g$ is called implemented by disclosure rule $\boldsymbol{\beta}$ if there is an externally enforced contract $m$ such that $m$ and $\beta$ implement $g$. Further, $g$ is called implementable if there exists an $m$ and $\beta$ that implement $g$.

Although this definition is written as "weak implementation," the strong sense is implied because the evidence-disclosure game is zero-sum and so all equilibria are equivalent.

The players' contractual goal is to implement a transfer function of their choice. We study the contracting problem by characterizing the set of implementable transfer functions.

\footnotetext{
${ }^{5}$ For simplicity, we focus here on pure disclosure rules. Our 2001 working paper extends the analysis to mixed rules (where players submit documents with nondegenerate probabilities); results are unchanged.

${ }^{6}$ Note that an individual player's behavior can easily be deduced from $\beta(a)$ since the players' document spaces are disjoint.
} 


\section{Interpretation}

Our model associates the common notion of "verifiability" with the court's observation of actual documents disclosed by the players. We use the word "documents" broadly, to include any testimony, statements, objects, etc. that can be submitted as evidence. As an example, consider a relationship between a creditor and a debtor. Suppose there are two states, one representing that the debtor has paid the creditor and the other representing that no payment was made. Three documents can be presented by the debtor. The first, denoted $d_{d}^{1}$, is a receipt given to the debtor by the creditor certifying payment. The second, $d_{d}^{2}$, is a canceled check that exists only if payment was made. The third document, $d_{d}^{3}$, is written testimony of payment made by the debtor's friend, who can be brought forth as a witness. Two documents may be presented by the creditor. The first, $d_{c}^{1}$, is a bank notice demonstrating that the debtor's check was returned due to insufficient funds. The second, $d_{c}^{2}$, is testimony of non-payment made by the creditor's colleague, who can be brought forth as a witness.

Figure 1 below describes how the availability of these documents depends on the state - that is, the specification of $D_{i}(a)$ sets - in this example. In the figure, an X indicates that a particular document exists and can be freely disclosed in a given state. For example, the debtor can produce a canceled check (document $d_{d}^{2}$ ) or a receipt (document $d_{d}^{1}$ ) only if she actually paid the creditor. However, it may be that she can get her friend to testify (document $d_{d}^{3}$ ) regardless of whether she paid. Similarly, the creditor can only produce the bounced check (document $d_{c}^{1}$ ) if the debtor did not pay, but the creditor can always find a witness to say that payment has not occurred (document $d_{c}^{2}$ ).

We use the term cheap document for any document that is available in every state. In the above example, $d_{d}^{3}$ and $d_{c}^{2}$ are both cheap. We refer to all non-cheap documents as hard evidence. We say that two documents of a single player are redundant if they exist in exactly the same states. For instance, $d_{d}^{1}$ and $d_{d}^{2}$ are redundant. Likewise, we say that two document sets for a player are redundant if they exist in exactly the same states; sets $\left\{d_{d}^{1}, d_{d}^{3}\right\}$ and $\left\{d_{d}^{2}, d_{d}^{3}\right\}$ have this property in the example.

In many contracting models (such as Legros and Matthews 1993 and Bernheim and Whinston 1999), verifiability is represented as a partition of the state space - a partition that is defined by the technological setting. Note that there is no such partition in our model, since what can be deduced about the state from disclosed documents depends on the players' disclosure rule. It can be helpful, though, to

\begin{tabular}{c|c|c|c|c|c|}
\multicolumn{1}{c}{} & \multicolumn{1}{c}{$d_{d}{ }^{1}$} & $d_{d}{ }^{2}$ & $d_{d}{ }^{3}$ & $d_{c}{ }^{1}$ & $d_{c}{ }^{2}$ \\
\cline { 2 - 6 } Paid & $\mathrm{X}$ & $\mathrm{X}$ & $\mathrm{X}$ & & $\mathrm{X}$ \\
\cline { 2 - 6 } Did not pay & & & $\mathrm{X}$ & $\mathrm{X}$ & $\mathrm{X}$ \\
\hline
\end{tabular}

Figure 1: Description of $D_{i}(a)$ sets in the debtor/creditor example. 
keep in mind how the evidence environment (characterized by the sets $D_{i}(a)$ ) and disclosure rules relate to partitions of the state space. We can start by calculating the set of states under which the same evidence set $E$ is produced by the players. Formally, if we know the players' disclosure rule $\beta$ and we observe evidence set $E$, where $E=\beta\left(a^{\prime}\right)$ for some state $a^{\prime}$, then we can deduce that the state is in the set $\{a \in A \mid \beta(a)=E\}$. Thus, $\beta$ suggests a partition of the state space given by

$$
\left\{\left\{a \in A \mid \beta(a)=\beta\left(a^{\prime}\right)\right\} \mid a^{\prime} \in A\right\} .
$$

This partition only reflects what can be deduced from the disclosure rule; it is obviously not a fundamental of the players' contracting problem. The disclosure rule itself is subject to availability of documents (from sets $D_{i}(a)$ ) as well as the players' incentives given the externally enforced contract $m$. The fineness of the induced partition corresponds to the extent that the players disclose distinct sets of evidence in different states.

\section{Technical Notes}

In our model, the "public decision" (what the court compels) is a transfer between the contracting parties, whereas, in more general models, the public decision could have any form of payoff relevance. We study transfers because we believe this is an accurate representation of external enforcement in many contractual relationships. Often, productive actions (such as investments and trading decisions) take place before the court intervenes; that is, remediation follows breach. Furthermore, even when there is some scope for courts to command productive activity, courts tend to be reluctant to force disputing parties back into a relationship by ordering specific performance. ${ }^{7}$ One may view the function $m$ as specifying liquidated damages for each contingency.

We could allow the players to write contracts that have them throwing away money (or transferring it to, say, a charity). This would be represented by $m_{1}+m_{2}<0$. However, this possibility would have no effect on the players' contractual goals if we assume that they can renegotiate between periods 3 and 4 , which seems realistic. If, after submitting evidence $E$, it is the case that $m_{1}(E)+m_{2}(E)<0$, then the players would renegotiate to some $m^{\prime}$ satisfying $m_{1}^{\prime}(E)+m_{2}^{\prime}(E)=0{ }^{8}$

Our model is essentially a static mechanism-design model that has two constraints. First, there is a fixed set of messages - the players' evidentiary decisions. Second, each player's set of feasible messages depends on the state. ${ }^{9}$ These features hinder

\footnotetext{
${ }^{7}$ This is not to say that messages or evidence production plays no role in the middle of an ongoing productive relationship. Our model focuses on evidence production at the time of external enforcement. One can include other messages in the specification of states, and study them separately.

${ }^{8} \mathrm{We}$ assume free renegotiation here. For a discussion of costly renegotiation, see Schwartz and Watson (2001) and Brennan and Watson (2002).

${ }^{9} \mathrm{~A}$ more general way of thinking about this is that players can present any particular document at a cost that depends on the state. We assume that, given $a$, documents in $D(a)$ can be disclosed at zero cost, while documents outside of this set can be produced only at infinite - or sufficiently prohibitive - cost. Bull (2001) contains preliminary analysis of moderate evidence-disclosure costs.
} 
the employment of standard mechanism-design methods and they can invalidate the revelation principle. Also, it may not be obvious whether or how one can translate the evidentiary structure into a direct-revelation form. Bull and Watson (2002) show that, under conditions satisfied here, a model with message constraints can be translated into an "abstract-declaration" model to which the revelation principle applies. In the abstract-declaration model-first developed and analyzed by Green and Laffont (1986) - the players simultaneously declare the state but are constrained in what they can say. Green and Laffont (1986) and Bull and Watson (2002) are concerned with translating between different modeling forms and the revelation principle. The characterization theorems presented here are stronger and follow from the added structure that we have assumed - in particular, that the court only compels transfers, which affect utility in an additive fashion.

\section{Characterization Results}

In this section, we study the set of implementable transfer functions. Our characterization results mainly concern (i) how the set of implementable transfer functions depends on the evidentiary structure, and (ii) whether implementation relies on particular documents being disclosed or withheld when they exist.

\section{Positive Evidence and Maximal Disclosure}

We start by characterizing how implementability depends on hard evidence and, further, how it is sensitive to the form of the hard evidence. Specifically, we differentiate between "positive" and "negative" evidence. ${ }^{10}$ Suppose there is a document $d$ that can be presented in state $a$ (that is $d \in D(a)$ ) but that does not exist in state $b$ $(d \notin D(b))$. Then disclosure of $d$ is considered positive evidence of $a$ since a player can present this document in state $a$ but not in some other state. Further, nondisclosure of $d$ is considered negative evidence of $b$ because a player cannot disclose $d$ in state $b$ but can disclose the document in some other state. For example, consider the canceled check in the debtor/creditor story. When the canceled check is presented, it is positive evidence that payment has been made. However, when the canceled check is not presented, this is negative evidence that payment has not been made.

Definition 3: We say that player $\boldsymbol{i}$ can distinguish between $\boldsymbol{a}$ and $\boldsymbol{b}$ if and only if $D_{i}(a) \neq D_{i}(b)$. We say that player $i$ can positively distinguish a from $\boldsymbol{b}$ if $D_{i}(a) \not \subset D_{i}(b)$.

To generate intuition regarding the connection between hard evidence and implementability, consider a case in which neither player can distinguish between states $a$ and $b$. That is, $D(a)=D(b)$. For such a setting, note that the evidence-disclosure game that is played in state $a$ is identical to the implied game in state $b$, because

\footnotetext{
${ }^{10}$ Lipman and Seppi (1995) study similar notions. Saltzburg (1978) studies negative inferences resulting from the absence of evidence and contrasts this with positive evidence.
} 
these two games have the same strategy spaces and payoff functions. ${ }^{11}$ The only way of implementing different transfers in states $a$ and $b$ would be through a different equilibrium selection in these two states. However, since the evidence-disclosure game is zero-sum, all equilibria are equivalent. As a result, every implementable transfer function satisfies $g(a)=g(b)$.

Clearly, then, implementing different transfers across states requires hard evidence that the players can use to distinguish between the states. Cheap documents are of no benefit in this regard. Thus, in our model, hard evidence is the basis of verifiability.

Implementation is also constrained by the type of hard evidence available. For example, suppose that $D_{1}(b) \subset D_{1}(a)$ and $D_{2}(a) \subset D_{2}(b)$. That is, player 1 cannot positively distinguish $b$ from $a$, whereas player 2 cannot positively distinguish $a$ from $b$. In this case, any implementable transfer function must satisfy $g_{1}(a) \geq g_{1}(b)$ and, equivalently, $g_{2}(a) \leq g_{2}(b)$.

To see why this is true, suppose $m$ and $\beta$ implement $g$. Note that, in state $a$, player 1 can disclose $\beta_{1}(b)$, so equilibrium requires

$$
m_{1}(\beta(a)) \geq m_{1}\left(\beta_{1}(b) \cup \beta_{2}(a)\right) .
$$

In state $b$, player 2 can disclose $\beta_{2}(a)$, so equilibrium requires

$$
m_{2}(\beta(b)) \geq m_{2}\left(\beta_{1}(b) \cup \beta_{2}(a)\right),
$$

which, using $m_{2}=-m_{1}$, implies $m_{1}\left(\beta_{1}(b) \cup \beta_{2}(a)\right) \geq m_{1}(\beta(b))$. Combining these inequalities yields $m_{1}(\beta(a)) \geq m_{1}(\beta(b))$, meaning that $g_{1}(a) \geq g_{1}(b)$.

Our first theorem shows that implementability is completely characterized by the notion of positive evidence.

Theorem 1: A transfer function $g$ is implementable if and only if, for all $a, b \in A$, $g_{1}(a)>g_{1}(b)$ implies that either player 1 can positively distinguish a from $b$ or player 2 can positively distinguish $b$ from $a$, or both.

This theorem is proved, along with the next result, at the end of this subsection.

We next show that, to determine whether a transfer function is implementable, it is sufficient to focus attention on full disclosure, in which each player submits all of the documents in his possession.

Definition 4: The full disclosure rule $\overline{\boldsymbol{\beta}}$ is defined by $\bar{\beta}(a) \equiv D(a)$ for all $a \in A$. $A$ transfer function $g$ is called implemented with full disclosure if there exists an externally enforced contract $m$ such that $g$ is implemented by $m$ and $\bar{\beta}$.

The following result characterizes implementability in terms of the full disclosure rule.

Theorem 2: Every implementable transfer function can be implemented with full disclosure.

\footnotetext{
${ }^{11}$ That they have the same payoff functions follows from the fact that the court only compels transfers (defined by $m$ ) and that utility is additive in transfers.
} 
We emphasize that Theorem 2 is not a revelation-principle result. The revelation principle addresses whether, in a mechanism-design environment, one can restrict attention to a particular class of game forms (direct-revelation forms) and to a particular equilibrium strategy (truthful reporting). Although our theorem identifies a specific equilibrium strategy (full disclosure), it does not address the choice of game form; in fact, the players' action space (evidence sets) is fixed in our model. Furthermore, in our model, the players' actions have no intrinsic meaning.

One can prove a revelation principle for the setting that we study here. The key to such an exercise is translating a setting of real documents into one of abstract messages; see Bull and Watson (2002) on how this is done and on the relation to Green and Laffont (1986). Theorem 2 yields stronger implications for implementability than does the revelation principle; these stronger implications come from the particular setting that we study here, where the court compels transfers.

Proof of Theorems 1 and 2: The "sufficiency" direction of Theorem 1 ( $g$ implementable implies the condition on $g$ ) is proved in the paragraph preceding the statement of Theorem 1. We prove the "necessity" direction of Theorem 1, as well as Theorem 2 by construction. Suppose the condition on $D_{1}$ and $D_{2}$ holds for $g$. We will design an externally enforced contract $m$ such that $m$ and $\bar{\beta}$ implement $g$. First, we translate evidence sets into "state declarations" by defining, for every $E \in \mathcal{D}$ and each player $i$,

$$
\Lambda_{i}(E) \equiv\left\{a^{i} \mid a^{i} \text { satisfies } D_{i}\left(a^{i}\right) \subset E_{i} \text { and } E_{j} \subset D_{j}\left(a^{i}\right)\right\},
$$

where $j$ is the other player. Then, for $i=1,2$, we let $\alpha^{i}(E)$ be the state $a^{i}$ that maximizes $g_{i}\left(a^{i}\right)$ over the elements of $\Lambda_{i}(E)$. We define $m(E) \equiv\left[g\left(\alpha^{1}(E)\right)+g\left(\alpha^{2}(E)\right)\right] / 2$, for every $E \in \mathcal{D}$.

We claim that $g\left(\alpha^{i}(D(a))=g(a)\right.$ for every $a \in A$ and $i=1,2$, which means that $g$ would be implemented if full disclosure is an equilibrium in every state. Since $g$ is balanced, it is sufficient to look at player $i$ 's transfer. To prove the claim, consider any state $a$ and any player $i$. We first note that $a \in \Lambda_{i}(D(a))$. This implies that $g_{i}\left(\alpha^{i}(D(a))\right) \geq g_{i}(a)$. We next show that $g_{i}\left(\alpha^{i}(D(a))\right) \leq g_{i}(a)$, proving the claim. To this end, presume that $g_{i}\left(\alpha^{i}(D(a))\right)>g_{i}(a)$ and we will find a contradiction. By the definition of $\Lambda_{i}(E)$, we know that $D_{i}\left(\alpha^{i}(D(a))\right) \subset D_{i}(a)$ and $D_{j}(a) \subset D_{j}\left(\alpha^{i}(D(a))\right)$. In words, player $i$ cannot positively distinguish $\alpha^{i}(D(a))$ from $a$, whereas player $j$ cannot positively distinguish $a$ from $\alpha^{i}(D(a))$. Given the conditions of the sufficiency part of Theorem 1, this contradicts $g_{i}\left(\alpha^{i}(D(a))\right)>g_{i}(a)$.

Finally, we observe that $\bar{\beta}$ is an equilibrium disclosure rule with respect to $m$. Note that the set $\Lambda_{i}(E)$ is increasing in $E_{i}$ and decreasing in $E_{j}$, for each player $i$. By the definition of $m$, full disclosure is a weakly dominant strategy for both players. Q.E.D. 


\section{Minimal Disclosure}

Theorem 2 is more of technical value than of practical relevance. In reality, it would be quite unusual for all available documents to be disclosed. Theorem 2 is not inconsistent with this observation, however; the theorem merely states that it is sufficient to examine implementation with full disclosure, not that implementation relies on full disclosure. For example, since cheap documents play no role in implementation here, they can be disclosed or left out. In this subsection, we characterize minimal sets of documents that are required for implementation.

Given any set $\underline{D} \subset D$, we define $\underline{D}_{i}(a) \equiv D_{i}(a) \cap \underline{D}$, for $i=1,2$ and every $a \in A$.

Definition 5: Take as given any set $\underline{D} \subset D$. We say that transfer function $\boldsymbol{g}$ is implementable on $\underline{\boldsymbol{D}}$ if there is a disclosure rule $\beta$ such that (i) $g$ is implemented by $\beta$ and (ii) $\beta(a) \subset \underline{D}$ for every $a \in A$.

We are interested in subsets of the document space that share some of the features of $D$.

Definition 6: Take as given any $\underline{D} \subset D$. We say that $\underline{\boldsymbol{D}}$ has the breadth property if, for all $a, b \in A$, (i) below implies (ii) below.

(i) $D_{1}(a) \not \subset D_{1}(b)$ or $D_{2}(b) \not \subset D_{2}(a)$ or both.

(ii) $\underline{D}_{1}(a) \not \subset \underline{D}_{1}(b)$ or $\underline{D}_{2}(b) \not \subset \underline{D}_{2}(a)$ or both.

If $\underline{D}$ has the breadth property, then $\underline{D}(a)$ represents the same state-distinctions that are implied by the full space of documents.

Our next result show that the breadth property is sufficient for implementability.

Theorem 3: The following is true for any document set $\underline{D}$ that has the breadth property. A transfer function $g$ is implementable if and only if it can be implemented on $\underline{D}$. Furthermore, if a document set $\underline{D}^{\prime}$ does not have the breadth property, then there is an implementable transfer function that cannot be implemented on $\underline{D}^{\prime}$.

This theorem is proved in Appendix A. The intuition is that sets with the breadth property sufficiently capture the degree to which the players can positively distinguish states from one another, which is the key to implementation as indicated in Theorem 1.

Theorem 3 establishes that the smallest document sets sufficient for implementation are those that are minimal with respect to the breadth property. A set $\underline{D}$ is minimal in this way if $\underline{D}$ has the breadth property and there is no other set $\underline{D}^{\prime} \subset \underline{D}$ such that $\underline{D}^{\prime} \neq \underline{D}$ and $\underline{D}^{\prime}$ has the breadth property.

Minimal sets are not unique. To see this, consider the example described in Figure 2(a). The row labelled " $D(\cdot)$ " shows the evidentiary structure. We assume that all of the documents are player 1's, so $D_{2}=\emptyset$. There are three minimal document sets in this example. These are shown as $\underline{D}^{A}, \underline{D}^{B}$, and $\underline{D}^{C}$ in the lowest three rows. 


\begin{tabular}{c|c|c|c|c|}
\multicolumn{5}{c}{ States } \\
\multicolumn{1}{c}{$a$} & \multicolumn{1}{c}{$c$} & \multicolumn{1}{c}{$c$} \\
\cline { 2 - 5 }$D()$. & $d^{1}, d^{4}, d^{6}$ & $d^{1}, d^{2}, d^{5}$ & $d^{2}, d^{3}, d^{6}$ & $d^{3}, d^{4}, d^{5}$ \\
\cline { 2 - 5 }$\underline{D}^{A}()$. & $d^{1}, d^{4}$ & $d^{1}, d^{2}$ & $d^{2}, d^{3}$ & $d^{3}, d^{4}$ \\
\cline { 2 - 5 }$\underline{D}^{B}()$. & $d^{1}, d^{6}$ & $d^{1}, d^{5}$ & $d^{3}, d^{6}$ & $d^{3}, d^{5}$ \\
\cline { 2 - 5 }$\underline{D}^{C}()$. & $d^{4}, d^{6}$ & $d^{2}, d^{5}$ & $d^{2}, d^{6}$ & $d^{4}, d^{5}$ \\
\cline { 2 - 5 } & & \multicolumn{3}{c}{}
\end{tabular}

(a)

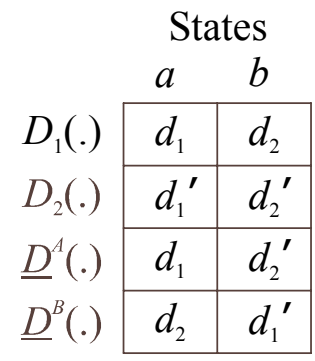

(b)

Figure 2: Description of $D$ and $\underline{D}$ in minimal disclosure rule examples.

Note that $\underline{D}^{A}=\left\{d^{1}, d^{2}, d^{3}, d^{4}\right\}, \underline{D}^{B}=\left\{d^{1}, d^{3}, d^{5}, d^{6}\right\}$, and $\underline{D}^{C}=\left\{d^{2}, d^{4}, d^{5}, d^{6}\right\}$, so none of these is a subset of another. One can easily verify that each of these has the breadth property and that all are minimal.

Figure 2(b) shows an example in which multiple minimal sets arise because of different ways of distinguishing between states across players. Here, both players can positively distinguish both states. In a minimal set, only one of the players' documents is needed. Thus, both $\underline{D}^{A}$ and $\underline{D}^{B}$ are minimal sets.

It is easy to see that minimal document sets never contain cheap documents. A cheap document does not influence the relationship between $D(a)$ and $D(b)$ for any $a, b \in A$. Further, a minimal document set will not contain two redundant document sets, because one can be removed while maintaining the breadth property.

\section{Partitions and Implementability}

In this subsection, we relate the set of implementable transfer functions to two partitions of the state space. For an arbitrary partition $P$ of the state space, and for any state $a$, we let $P(a)$ denote the element of the partition that contains $a$. That is, $b \in P(a)$ if and only if $a$ and $b$ are in the same element of the partition $P$. We denote by $P^{D}$ the partition of $A$ that is induced by the notion of distinguishing between states. Formally $P^{D}$ is defined so that $a \in P^{D}(b)$ if and only if $D(a)=D(b)$.

We say that player $i$ can fully distinguish between states $a$ and $b$ if neither $D_{i}(a) \subset$ $D_{i}(b)$ nor $D_{i}(b) \subset D_{i}(a)$. To fully distinguish between $a$ and $b$, a player must have positive evidence of $a$, to the exclusion of $b$, as well as positive evidence of $b$, to the exclusion of $a$. For either player $i$, we define the partition $\bar{P}^{D_{i}}$ as the finest partition satisfying

$$
D_{i}(a) \subset D_{i}(b) \text { implies } a \in \bar{P}^{D_{i}}(b) .
$$

This partition reflects the notion of player $i$ fully distinguishing between states, which the next result associates with a lower bound on the set of implementable transfer functions.

Theorem 4: Every implementable transfer function is measurable with respect to $P^{D}$. Furthermore, for $i \in\{1,2\}$, every transfer function that is measurable with respect to $\bar{P}^{D_{i}}$ is implementable. 
As Theorem 4 shows, if a player can fully distinguish between states then a contract exists that essentially forces her to disclose evidence that isolates the actual state from the others. This is accomplished by severely punishing the player for not disclosing one of these critical documents.

\section{Legal Applications}

In this section, we use our model to comment on two issues regarding the functioning of actual legal institutions.

\section{The Meaning and Types of Evidence}

The law defines evidence as "testimony, writings, material objects, or other things presented to the senses that are offered to prove the existence or nonexistence of a fact." ${ }^{12}$ Evidence is admissible only if it is relevant - that is, only if it serves to prove or disprove a fact of consequence. ${ }^{13}$ Our model of evidence is consistent with this legal view, because a disclosure rule and evidentiary constraints make evidence informative on the equilibrium path. In other words, a third party can infer something about the state by observing the documents that the players disclose (as described in Section 1). Our model demonstrates the importance of hard evidence in settings in which the court compels transfers.

Beyond the issue of relevance, the legal system recognizes the difference between positive and negative evidence. Our theory identifies positive distinctions as the strongest form of evidence, because, once hard evidence is disclosed, it rules out some states regardless of the players' strategic intentions. In fact, the law is broadly consistent with this stance. Positive evidence presented to substantiate a claim is always given weight by the legal system. Further, although U.S. courts recognize the significance of negative evidence, they generally treat it as less compelling than is positive evidence. Wigmore (1935) suggests that failure by a party to disclose evidence that would be favorable to her claim if it existed is "open to the inference that it does not exist." But the negative inference depends on the party's incentives. Thus, Wigmore (1940) notes that uncertainty exists as to the exact "nature of the inference and conditions in which it may legitimately be drawn." For example, consider the two player, two state example sketched in the Introduction. Suppose it is known that a single document $d$ is available to player 2 in state $a$ but does not exist in state $b$. As suggested, a contract can be structured to give player 2 the incentive to disclose $d$ when this document is available. Thus, under such a contract (and assuming rational behavior), her failure to disclose $d$ can be taken to mean that $d$ does not exist. However, this conclusion may not be reached if the original contract

\footnotetext{
${ }^{12}$ California Evidence Code $\S 140$. The Federal Rules of Evidence does not define evidence formally. Classic legal treatments of evidence are Bentham (1827) and Wigmore (1940). See also Posner (1999).

${ }^{13}$ We are paraphrasing Federal Rules of Evidence 401, which is similar to C.E.C. $§ 210$.
} 
failed to provide player 2 with the incentive to disclose $d .{ }^{14}$

An unrealistic feature of our model (and of mechanism-design models in general) is that it assumes the court will enforce any contracted mapping from evidence to transfers. In reality, however, the legal system imposes constraints on this mapping. In fact, in practice, the actual mapping from evidence to public decisions is a composite function. Contracts usually specify what the parties are supposed to do and, perhaps, liquidated damages. Then the court assesses the state and compels remedies. Legal constraints enter through the application of evidentiary rules (on direct and circumstantial evidence, burdens of proof, presumptions, and so on) and through the application of rules of law to the facts of the case (for example, breach remedies). Although our current modeling exercise does not directly analyze these constraints, variants of our model may be of some use in this regard. ${ }^{15}$ Two related papers, which analyze rules of law, are Sanchirico (1999) and Bernardo, Talley, and Welch (2000). ${ }^{16}$

One type of legal constraint is partly addressed by our model: that relevance is not always sufficient for the admission of evidence. For example, relevant evidence is sometimes excluded if its probative value is outweighed by the danger of unfair prejudice, confusion, or waste of time. ${ }^{17}$ Although we do not model the possible detrimental effects of allowing such evidence, we do show that some types of evidence - cheap and redundant documents, for instance - are not needed for implementation. Specifically, Theorem 3 provides some insight as to what evidence can be excluded without limiting implementability.

\section{Enforced Discovery}

The process of discovery, which is institutionalized in U.S. law, allows parties to request evidence from one another. In terms of our model, it would be like player 1 declaring, "Player 2, I know you have document $d$; I request that you disclose it." In reality, the law tries to enforce discovery requests. If it were possible for the law to

\footnotetext{
${ }^{14}$ A classic case, State v. Simons $(1845)$ provides a good example in which the court's decision is based on negative evidence. Simons was accused of selling spirits without a license. The court held that the failure of Simons to present a license, even though State presented no evidence showing lack of a license, was sufficient to conclude that he did not possess such license. The court's opinion suggests that evidence "need not be of the most direct and positive kind."

${ }^{15}$ It may be interesting to consider the differences between various presumption rules (such as the Thayer-Wigmore theory and F.R.E. 301) under some fixed assumptions about the relation between states and the players' actions.

${ }^{16}$ Sanchirico (1999) studies, in a tort setting, the relation between the level of care and the cost of providing evidence. The evidentiary costs are state-dependent, evidence can be forged, and the court's decision is a fixed function of evidence. Bernardo, Talley, and Welch (2000) study legal presumptions using a principal-agent model with the possibility of litigation following a lowproduction outcome. The agent's cost of evidence production depends on her level of productive effort. In some models which view the court as a Bayesian decisionmaker, such as Sobel (1985) and Shin (1994 and 1998), the decisionmaker is allowed to reallocate the burden of proof.

${ }^{17}$ See F.R.E. 402, 403. Further, there are exclusionary legal rules that are not rules of evidence, such as the Fourth Amendment rule excluding the products of illegal search and seizure, the federal law prohibiting wiretaps, the work product doctrine, and the parole evidence rule.
} 
enforce every legitimate request then, effectively, the players could always force each other to disclose existing documents. We next show how this strong form of enforced discovery can be represented in our model and what it implies for implementability. We then discuss why it is typically not possible.

We call the contractual setting one of enforced discovery requests if each player can force the other player to disclose existing documents. Since our model assumes disjoint document spaces, we represent enforced discovery by assuming that each player has copies of each other's documents. Mathematically, this means that for $i, j \in\{1,2\}$ and each document $d \in D_{i}$, there exists a document $d^{\prime} \in D_{j}$ such that $d \in D_{i}(a)$ if and only if $d^{\prime} \in D_{j}(a)$. In words, if one player can positively distinguish $a$ from $b$, then the other player can also do so.

With enforced discovery requests, the set of implementable transfer functions is not constrained by the failure of positive evidence.

Theorem 5 In the setting of enforced discovery requests, the difference between positive and negative evidence is not critical, and any transfer function that is measurable with respect to $P^{D}$ is implementable.

The intuition behind this result is that, if one player wants to suppress a document that lowers her transfer, then the other player will benefit from the document being disclosed and, thus, will disclose the copy.

As Theorem 5 establishes, enforced discovery makes any form of hard evidence sufficient for implementability. However, enforced discovery is not often possible in reality. Since the court does not observe the state, it cannot differentiate between (i) the failure of a player to honor a discovery request and (ii) an illegitimate request (such as player 1 asking player 2 to disclose $d$ when $d$ does not exist). That is, verification of compliance with a discovery request is tantamount to verification of the state. Our fundamental assumption is that the court cannot directly verify the state, so neither can the court enforce discovery requests. As Brazil (1978) and Shapiro (1979) suggest, in practice the discovery process does not result in the intended open exchange of information because of the parties strategic interests (suppressing evidence and making illegitimate requests).

Courts may be able to offer limited enforcement of discovery requests in some cases. For example, the court may know that a particular class of documents always exists and, thus, may be able to force a player to disclose at least one document from this class. However, the effect of enforced discovery in this case could be achieved by the players' initial contract, so enforced discovery adds little to our theoretical understanding. Still, there may be a practical distinction between enforced discovery and an effective contract. Furthermore, disclosure costs may play an important role in reality. Along this line, Cooter and Rubinfeld (1994), in a setting of costly evidence production, characterize an efficient level of discovery requests, while Cooter and Rubinfeld (1995) show that current federal law does not adequately prevent the problem of excessive discovery requests. Bull (2001) provides a preliminary analysis of evidence production costs in a variant of our model. 


\section{Settings With More Than Two Players}

In this section, we extend our analysis to settings with $n$ players, where $n \geq 2$. Our main objective is to identify conditions under which versions of Theorems 1-5 are valid. In particular, we seek to understand when verifiability is characterized in terms of hard evidence. This requires more stringent conditions on behavior in period 3 than we assumed for the two-player case. Specifically, we add a side-contracting condition.

\section{Definitions}

The $n$-player model has the same timing as does our basic model. The definitions and notation are also the same, except now we have player indices from 1 to $n$. Thus, $D_{1}, D_{2}, \ldots, D_{n}$ are the players' document spaces, assumed disjoint. The externallyenforced contract is a function $m: \mathcal{D} \rightarrow \mathbf{R}_{0}^{n}$. A transfer function is given by $g$ : $A \rightarrow \mathbf{R}_{0}^{n}$. We assume that the transfers are balanced for the same reason that we discussed in Section 1. We let $N \equiv\{1,2, \ldots, n\}$ and, for any $J \subset N$, we define $\beta_{J}(a) \equiv \bigcup_{i \in J} \beta_{i}(a)$.

As with the two-player model, we analyze behavior in period 3 using the standard Nash equilibrium concept. Thus, given $m$, we call $\beta$ an equilibrium disclosure rule if

$$
m_{i}(\beta(a)) \geq m_{i}\left(E_{i} \cup \beta_{-i}(a)\right)
$$

for each $i \in N, E_{i} \subset D_{i}(a)$, and $a \in A$. In addition to equilibrium, however, we add constraints reflecting the players' ability to make side deals between periods 2 and 3 . In particular, we shall impose a version of Bernheim, Peleg, and Whinston's (1987) coalition-proofness concept that assesses whether a sub-group of players can benefit from side contracting. We examine whether a coalition can gain from writing a side contract $m^{\prime}$ to be externally enforced along with the players' original contract $m$.

To illustrate our side-deal constraint, suppose that a coalition of players $J \subset N$ wishes to write an additional contract $m^{\prime}$ specifying transfers between members of this coalition as a function of the documents disclosed in period 3 . It must be that $m_{i}^{\prime}=0$ for each $i \neq J$, because coalition $J$ cannot force a side contract on players outside of the coalition. In addition, $m^{\prime}$ must be balanced due to the specter of renegotiation between periods 3 and 4 . We assume that $m^{\prime}$ is enforced, either by the court or by some other means. With the side contract, externally enforced transfers in period 4 are given by $m+m^{\prime}$.

We assume that players outside the coalition do not observe the side contract until after documents are disclosed. Thus, the point of writing a side contract is to induce members of the coalition to change their behavior in the evidence-disclosure game in a way that benefits the coalition. To evaluate whether this is possible, define $M_{J}(E) \equiv \sum_{j \in J} m_{j}(E)$ and let $M_{J}^{\prime}(E)$ be defined analogously. Suppose that in state $a$ the players would coordinate on disclosure of documents $\beta(a)$ in the absence of side contracting. Further suppose that, by side contracting, a coalition $J$ can induce its members to disclose documents $E_{J}$. Then the coalition strictly gains from the side 
deal if and only if

$$
M_{J}\left(\beta_{-J}(a) \cup E_{J}\right)+M_{J}^{\prime}\left(\beta_{-J}(a) \cup E_{J}\right)>M_{J}(\beta(a))+M_{J}^{\prime}(\beta(a)),
$$

which is equivalent to $M_{J}\left(\beta_{-J}(a) \cup E_{J}\right)>M_{J}(\beta(a))$ because $M_{J}^{\prime}=0$. Here, " $-J$ " stands for the complement of $J$.

Between periods 2 and 3, a $J$-coalition can always find a side contract that induces its members to disclose any particular set of documents, as long as the specified documents exist given the state. This is because the coalition writes a side contract after players observe the state-knowing which documents exist. The coalition can write a forcing side contract that punishes individuals for not disclosing exactly those documents desired by the coalition (by way of arbitrarily large transfers to the other members of the coalition). The side contract can also implement any desired split of its gains between the coalition members. Thus, between periods 2 and 3, coalitions can effectively spot contract on which documents to disclose. Mathematically, in state $a$, a coalition $J$ can side contract to force its members to disclose any set of documents $E_{J} \subset D_{J}(a) \equiv \bigcup_{j \in J} D_{j}(a)$.

In practical terms, a side contract may amount to joint disclosure of documents, which is generally possible in reality. ${ }^{18}$ Mathematically, however, we are assuming a greater scope for side deals. Specifically, side contracts may induce players to withhold documents that they might otherwise disclose. Furthermore, side contracts can be used by a coalition to rearrange transfers internally. While there are real examples of private parties that enforce side contracts, we acknowledge that our modeling of side deals imposes stronger constraints on implementability than probably exist in most contractual relationships.

A side contract between members of a coalition $J$ may be undermined by a subsequent side contract between members of a sub-coalition $K \subset J$. Following Bernheim, Peleg, and Whinston (1987), we view a side contract as viable only if it is immune to disruption by sub-coalitions (who have to pass the same test). In fact, the issue of sub-coalitions is easily handled in our model, because forcing contracts can always be designed to stifle any further side dealing by sub-coalitions. Specifically, a coalition $J$ can specify $m^{\prime}$ so that any player $j \in J$ who does not disclose a specified set of documents must pay an amount $y$ to each of the other players in the coalition. Then any sub-coalition $K \subset J$ will lose at least $y$ when one or more of its members deviates from the prescription of $m^{\prime}$. The number $y$ can be set large enough so that this loss is greater than any gain the sub-coalition can get by way of the original contract $m$.

We look for specifications of behavior that are coalition-proof with respect to externally enforced side contracts. Given the discussion above, it is sufficient to simply evaluate whether coalitions can gain from spot contracting on disclosure of documents. With reference to a state $a$ and a disclosure rule $\beta$, we say that $E \in \mathcal{D}$ is a $J$-deviation from $\beta(a)$ at $a$ if $E \subset D(a)$ and $E_{i}=\beta_{i}(a)$ for all $i \notin J$. In words, $E$ is a set of documents disclosed when only the players in $J$ deviate from the prescription $\beta(a)$. Coalition-proofness is defined as follows.

\footnotetext{
${ }^{18}$ For a discussion of civil procedure, see, for example, Teply, L. and R. Whitten (2000).
} 
Definition 7: Given an externally enforced contract $m$, a disclosure rule $\beta$ is called impervious to side contracting (ISC) if $M_{J}(\beta(a)) \geq M_{J}(E)$ for each a $\in A$, each coalition $J \subset N$, and each $E \subset D(a)$ that is a $J$-deviation from $\beta(a)$ at a.

We use the term impervious to side contracting instead of coalition-proof Nash equilibrium because the latter is defined for self-enforced contracts (Nash equilibria) of standard non-cooperative games, while we require a version that examines externally enforced contracts. That is, ISC is Bernheim, Peleg, and Whinston's (1987) definition applied to externally enforced contracts. Note that the ISC condition includes deviations by a single player, so every ISC disclosure rule is also an equilibrium disclosure rule. ISC coincides with equilibrium in the case of $n=2$.

Definition 8: We say that a transfer function $g$ is implemented by an ISC disclosure rule if there are functions $m$ and $\beta$ such that (i) $\beta$ is an ISC disclosure rule with respect to $m$, and (ii) $g(a)=m(\beta(a))$ for every $a \in A$.

Definition 9: A transfer function $g$ is called implemented by an ISC/full disclosure rule if there exists an externally enforced contract $m$ such that (i) the full disclosure rule $\bar{\beta}$ is ISC with respect to $m$, and (ii) $g$ is implemented by $m$ and $\bar{\beta}$.

\section{A Partial Generalization of the Results}

We offer the following extensions of Theorems 1, 2, and 4. The proofs of these results are in Appendix A.

Theorem 1': If transfer function $g$ is implemented by an ISC disclosure rule then, for every subset of players $J \subset N$ and every pair of states a and $b, G_{J}(a)>G_{J}(b)$ implies that either a player in group $J$ can positively distinguish a from $b$ or a player in group $-J$ can positively distinguish $b$ from $a$, or both.

Here $G_{J}$ means the sum of transfers to the players in the $J$ group; that is, $G_{J}(a) \equiv$ $\sum_{i \in J} g_{i}(a)$.

Theorem 2': If a transfer function $g$ is implemented by an ISC disclosure rule, then $g$ is implemented by an ISC/full disclosure rule.

Theorem 4': Every implementable transfer function is measurable with respect to $P^{D}$. Furthermore, for any $i \in N$, every transfer function that is measurable with respect to $\bar{P}^{D_{i}}$ is implementable.

Theorem 5 holds in the $n$-player setting without modification. The proof presented in Appendix A is valid for two or more players.

These theorems show that cheap documents are ineffective in the $n$-player setting (with the ISC condition), just as they are in the two-player environment. That is, implementability is determined by hard evidence. In mechanism-design parlance, this means that "message game phenomena" do not exist. In other words, endowing the players with the ability to freely communicate with the court (by sending 
any number of cheap documents) does not enlarge the set of implementable transfer functions beyond what could be achieved using hard evidence. Clearly, this result is due to the combination of the ISC condition and the zero-sum aspect of externally enforced transfers. The conclusion does not necessarily hold for settings in which some productive actions are taken after documented messages are sent, since then continuation payoffs may not be zero-sum. Yet our results do demonstrate that, in general, the opportunity for players to side-contract can significantly limit message game phenomena.

Note that Theorem $1^{\prime}$ is only a partial generalization of Theorem 1 , because only the "sufficiency" direction is stated. In fact, the "necessity" direction does not hold generally, as the following counterexample demonstrates. Suppose there are three states, $A=\{a, b, c\}$, and three players. In state $a$, player 1 has no documents, player 2 has document $d_{2}^{a}$, and player 3 has document $d_{3}^{a}$. In state $b$, player 1 has $d_{1}^{b}$, player 2 has nothing, and player 3 has $d_{3}^{b}$. In state $c$, player 1 has $d_{1}^{c}$, player 2 has $d_{2}^{c}$, and player 3 has nothing. Note that player 1 can fully distinguish between $b$ and $c$, player 2 can fully distinguish between $a$ and $c$, and player 3 can fully distinguish between $a$ and $b$.

For every pair of states and for every subset $J$ of players, either a player in $J$ or a player in the complement of $J$ can fully distinguish between the pair. This means that the condition on positive distinctions in Theorem $1^{\prime}$ is satisfied for every transfer function. Consider function $g$ defined by $g(a)=(2,-1,-1), g(b)=(-1,2,-1)$, and $g(c)=(-1,-1,2)$. For example, suppose $J=\{2,3\}$, for which we have $G_{J}(a)<$ $G_{J}(b)$. In this case, player 3 can positively distinguish $b$ from $a$, so the condition holds.

In fact, $g$ cannot be implemented. To see this, first note that, if $g$ is implementable, then it can be implemented with full disclosure. Therefore, we can assume that $m\left(\left\{d_{2}^{a}, d_{3}^{a}\right\}\right)=(2,-1,-1), m\left(\left\{d_{1}^{b}, d_{3}^{b}\right\}\right)=(-1,2,-1)$, and $m\left(\left\{d_{1}^{c}, d_{2}^{c}\right\}\right)=(-1,-1,2)$. Consider what happens when no document is disclosed, so the evidence set is $E=\emptyset$. Observe that players 2 and 3 can jointly deviate to $E$ in state $a$. Thus, the ISC condition requires $m_{2}(\emptyset)+m_{3}(\emptyset) \leq-2$; equivalently, $m_{1}(\emptyset) \geq 2$. Further, since players 1 and 3 can jointly deviate to $E$ in state $b$, we need $m_{2}(\emptyset) \geq 2$. Finally, since players 1 and 2 can jointly deviate to $E$ in state $c$, we need $m_{3}(\emptyset) \geq 2$. This yields a violation of balancedness, a contradiction.

\section{The Case of Complete Environments}

The counterexample of the previous subsection shows that, with more than two players, there are additional constraints on implementability than those implied by the lack of positive distinctions. The additional constraints are due to evidence sets that can be disclosed in more than one state but that do not correspond to full disclosure in any particular state. The set $\emptyset$ functions this way in the example. In the absence of such problematic evidence sets, we obtain the full generalized versions of Theorems 1 and 3 . 
Definition 10: We say that the state and evidence environment is complete if, for every evidence set $E \in \mathcal{D}$, there exists a state a such that $D(a)=E$.

Theorem 1": Assume the state and evidence environment is complete. A transfer function $g$ is implemented by an ISC disclosure rule if and only if, for every subset of players $J \subset N$ and every pair of states a and $b, G_{J}(a)>G_{J}(b)$ implies that either a player in group $J$ can positively distinguish a from $b$ or a player in group $-J$ can positively distinguish $b$ from $a$, or both.

To state the generalized version of Theorem 3 , we need to first generalize the breadth property.

Definition 6": Take as given any $\underline{D} \subset D$. We say that $\underline{\boldsymbol{D}}$ has the breadth property if, for all $a, b \in A$ and every set $J \subset N$, (i) below implies (ii) below.

(i) $D_{J}(a) \not \subset D_{J}(b)$ or $D_{-J}(b) \not \subset D_{-J}(a)$ or both.

(ii) $\underline{D}_{J}(a) \not \subset \underline{D}_{J}(b)$ or $\underline{D}_{-J}(b) \not \subset \underline{D}_{-J}(a)$ or both.

Theorem $3^{\prime \prime}$ : Assuming the state and evidence environment is complete, the following is true for any document set $\underline{D}$ that has the breadth property. A transfer function $g$ is implementable with ISC disclosure if and only if it can be implemented with ISC disclosure on $\underline{D}$. Furthermore, if a document set $\underline{D^{\prime}}$ does not have the breadth property, then there is a transfer function that is implementable with ISC disclosure but not on $\underline{D}^{\prime}$.

\section{Conclusion}

We have presented a model of contract and external enforcement in which implementation depends on the existence and form of hard evidence. Thus, "verifiability" is associated with hard evidence and its implications are qualified by the identity of the evidence holder and the form of the hard evidence. The modeling exercise reported here follows Watson's (2001) program of explicitly modeling inalienable decisions and technological constraints in contractual relations. Our model is, however, simplistic in its representation of actual evidence production. There are several promising directions for related research, some of which have been partly addressed in the recent literature: (a) analysis of intermediate evidence-production costs, (b) examining litigation procedures that involves sequential document disclosure, (c) studying settings in which the players have private information about available documents, and (d) adding more institutional details and comparing different litigation systems (for example, default rules, burden of proof considerations, and evidence admissibility rules). 


\section{A Proofs}

This appendix contains the analysis that is not reported in the main body of the paper. Theorems are restated here for the reader's convenience.

Theorem 3: The following is true for any document set $\underline{D}$ that has the breadth property. A transfer function $g$ is implementable if and only if it can be implemented on $\underline{D}$. Furthermore, if a document set $\underline{D}^{\prime}$ does not have the breadth property, then there is an implementable transfer function $g$ that cannot be implemented on $\underline{D}^{\prime}$.

Proof: By definition, a document set $\underline{D}$ that has the breadth property inherits from $D$ enough documents to provide the distinctions necessary for implementability, as described in Theorem 1 . Thus, one can restrict the players to $\underline{D}$ and preserve the conclusion of Theorem 1, which proves the first claim.

To prove the second claim, we note that, since $\underline{D}^{\prime}$ does not satisfy the breadth property, there exist states $a$ and $b$ such that $\underline{D}_{1}^{\prime}(a) \subset \underline{D}_{1}^{\prime}(b)$ and $\underline{D}_{2}^{\prime}(b) \subset \underline{D}_{2}^{\prime}(a)$, but either $D_{1}(a) \not \subset D_{1}(b)$ or $D_{2}(b) \not \subset D_{2}(a)$, or both.

Take the case in which $D_{1}(a) \not \subset D_{1}(b)$ and let $d^{\prime}$ be such that $d^{\prime} \in D_{1}(a)$ and $d^{\prime} \notin D_{1}(b)$. Define $g$ by $g(c)=(1,-1)$ for each $c \in A$ for which $d^{\prime} \in D(c)$, and $g(c)=(0,0)$ for each $c$ satisfying $d^{\prime} \notin D(c)$. Let $m$ be defined by $m(E)=(1,-1)$ if $d^{\prime} \in E$, and $m(E)=(0,0)$ otherwise. Clearly, player 1 has the incentive to disclose $d^{\prime}$ whenever it exists and so $g$ is implemented by $m$ and $\bar{\beta}$. However, $g$ cannot be implemented on $\underline{D}^{\prime}$. To see this, suppose that $g$ is implemented on $\underline{D}^{\prime}$ by contract $m^{\prime}$ and disclosure rule $\beta^{\prime}$. We know that $\beta_{1}^{\prime}(a) \subset \underline{D}_{1}^{\prime}(b)$ and $\beta_{2}^{\prime}(b) \subset \underline{D}_{2}^{\prime}(a)$. Equilibrium requires

$$
m_{1}^{\prime}\left(\beta^{\prime}(b)\right)=0 \geq m_{1}^{\prime}\left(\beta_{1}^{\prime}(a) \cup \beta_{2}^{\prime}(b)\right)
$$

and

$$
m_{2}^{\prime}\left(\beta^{\prime}(a)\right)=-1 \geq m_{2}^{\prime}\left(\beta_{1}^{\prime}(a) \cup \beta_{2}^{\prime}(b)\right) .
$$

Because $m_{1}^{\prime}(E)=-m_{2}^{\prime}(E)$ for all $E \in \mathcal{D}$, these inequalities yield a contradiction. The same type of construction works for the case of $D_{2}(b) \not \subset D_{2}(a)$. Q.E.D.

Theorem 4: Every implementable transfer function is measurable with respect to $P^{D}$. Furthermore, for $i \in\{1,2\}$, every transfer function that is measurable with respect to $\bar{P}^{D_{i}}$ is implementable.

Proof: Theorem 1 implies that measurability with respect to $P^{D}$ is necessary for implementability, proving the first claim. To prove the second claim, take any transfer function $g$ that is measurable with respect to $\bar{P}^{D_{i}}$. Consider a contract $m$ defined as follows. For each $a \in A$ and every $E_{j} \subset D_{j}(a)$, define $m_{i}\left(E_{i} \cup E_{j}\right)=g(a)$. For every evidence set $E$ such that

$$
E_{i} \notin\left\{E_{i} \mid E_{i}=D_{i}(a) \text { for some } a \in A\right\},
$$

define $m_{i}(E)=-m_{j}(E)=\mu$, where $\mu$ is any number satisfying $\mu<\min _{a \in A} g_{i}(a)$. Note that $g(a)=g(b)$ for those states $a$ and $b$ between which player $i$ cannot fully 
distinguish. Because $D_{i}\left(a^{\prime}\right) \not \subset D_{i}\left(b^{\prime}\right)$ and $D_{i}\left(b^{\prime}\right) \not \subset D_{i}\left(a^{\prime}\right)$ for all other states $a^{\prime}$ and $b^{\prime}$, player $i$ has the incentive to fully disclose in each state. The other player is indifferent between all of his evidentiary decisions. Thus, $g$ is implemented by $m$ and $\bar{\beta}$. Q.E.D.

From this point, our analysis concerns the general case of two or more players.

Theorem 5: In the setting of enforced discovery requests, the difference between positive and negative evidence is not critical, and any transfer function that is measurable with respect to $P^{D}$ is implementable.

Proof: We shall provide an argument that establishes the result in the general case of two or more players. Let $g$ be a transfer function that is measurable with respect to $P^{D}$. For each player $i>1$, there is a one-to-one mapping $\lambda_{i}: D_{i} \rightarrow D_{1}$ such that $d \in D_{i}(a)$ if and only if $\lambda_{i}(d) \in D_{1}(a)$. This follows from the assumption of enforced discovery requests. For example, suppose that, in state $a$, player 1 has document $d$. Then the other players have copies of $d$ identified by $\lambda_{2}^{-1}(d), \lambda_{3}^{-1}(d)$, and so on. This defines an equivalence class of documents, in which $d$ is equivalent to $\lambda_{i}^{-1}(d)$ for every $i>1$.

For each evidence set $E \in \mathcal{D}$, define

$$
\Gamma(E) \equiv E_{1} \cup \lambda_{2}\left(E_{2}\right) \cup \lambda_{3}\left(E_{3}\right) \cup \cdots \cup \lambda_{n}\left(E_{n}\right) .
$$

Then define $m$ as follows. For each $E \in \mathcal{D}$ such that $\Gamma(E)=D_{1}(a)$ for some $a \in A$, we specify $m(E)=g(a)$. For every other set $E, m(E)$ can be defined arbitrarily; $m(E)=(0,0, \ldots, 0)$ will do. The idea is that $m$ is defined on the equivalence classes of documents.

Observe that $\bar{\beta}$ is an ISC disclosure rule with respect to $m$ (in the two-player case, equilibrium). This is because no single player or coalition can gain by deviating in any state. For example, if player 1 withholds document $d$ in state $a$, it will have no effect on the transfer because each player $i \neq 1$ is disclosing the copy $\lambda_{i}^{-1}(d)$. Thus, $g$ is implemented by $m$ and $\bar{\beta}$. Q.E.D.

Theorem 1': If transfer function $g$ is implemented by an ISC disclosure rule then, for every subset of players $J \subset N$ and every pair of states $a$ and $b, G_{J}(a)>G_{J}(b)$ implies that either a player in group $J$ can positively distinguish $a$ from $b$ or a player in group $-J$ can positively distinguish $b$ from $a$, or both.

Proof: Suppose $m$ and $\beta$ implement $g$. It is sufficient for us to show that $D_{J}(a) \subset$ $D_{J}(b)$ and $D_{-J}(b) \subset D_{-J}(a)$ together imply $G_{J}(a) \leq G_{J}(b)$. Assume $D_{J}(a) \subset D_{J}(b)$ and $D_{-J}(b) \subset D_{-J}(a)$. Note that, in state $b$, coalition $J$ can disclose $\beta_{J}(a)$, so equilibrium requires

$$
M_{J}(\beta(b)) \geq M_{J}\left(\beta_{J}(a) \cup \beta_{-J}(b)\right) .
$$

In state $a$, coalition $-J$ can disclose $\beta_{-J}(b)$, so equilibrium requires

$$
M_{-J}(\beta(a)) \geq M_{-J}\left(\beta_{J}(a) \cup \beta_{-J}(b)\right),
$$


which, using $M_{J}=-M_{-J}$, implies $M_{J}\left(\beta_{J}(a) \cup \beta_{-J}(b)\right) \geq M_{J}(\beta(a))$. Combining these inequalities yields $M_{J}(\beta(b)) \geq M_{J}(\beta(a))$, meaning that $G_{J}(b) \geq G_{J}(a)$. Q.E.D.

Theorem 2': If a transfer function $g$ is implemented by an ISC disclosure rule, then $g$ is implemented by an ISC/full disclosure rule.

Proof: This proof is involved, but we find the construction quite interesting. It will be convenient for us to write some of the components of the proof as lemmas.

We begin the analysis with a simple characterization of the ISC condition. For each $a \in A, E \subset D(a)$, and $E^{\prime} \in \mathcal{D}$, we define the function $R\left(a ; E, E^{\prime}\right)$ as follows. If $E^{\prime} \not \subset D(a)$, then we let $R\left(a ; E, E^{\prime}\right) \equiv N$. If $E^{\prime} \subset D(a)$ then we let $R\left(a ; E, E^{\prime}\right) \equiv$ $\left\{i \in N \mid E_{i} \neq E_{i}^{\prime}\right\}$. That is, $R\left(a ; E, E^{\prime}\right)$ is the minimum set of players that would be needed to deviate from $E$ in order to achieve $E^{\prime}$ in state $a$.

Lemma 1: Given $m, \beta$ is an ISC disclosure rule if and only if $m_{i}(\beta(a)) \leq$ $m_{i}(E)$, for each $a \in A$, every $E \in \mathcal{D}$, and all $i \notin R(a ; \beta(a), E)$.

To prove this lemma, suppose that, for some equilibrium disclosure rule $\beta(a)$ and some state $a$, there is a set of documents $E$ and a player $i$ such that $i \notin R(a ; \beta(a), E)$ and $m_{i}(\beta(a))>m_{i}(E)$. This means that if the players in group $R(a ; \beta(a), E)$ achieve disclosure of $E$ by altering what documents they present, player $i$ is strictly worse off. Since $m$ is balanced, this implies that the other players $(-i)$ are collectively strictly better off when $E$ is disclosed rather than $\beta(a)$. Further, since $R(a ; \beta(a), E) \subset-i$ we know that $E$ is a $-i$-deviation from $\beta(a)$ at $a$, which means $\beta(a)$ could not be ISC. In the other direction the condition of the lemma obviously implies that no coalition can strictly gain by deviating from $\beta$. Thus, Lemma 1 is established.

Next we provide a necessary and sufficient condition for a transfer function $g$ to be implemented by an ISC disclosure rule, where we focus on a given disclosure rule $\beta$. The analysis examines the different ways in which coalitions of players can deviate to induce disclosure of an arbitrary set of documents. For example, consider some set of documents $E$ and states $a$ and $b$. Disclosure rule $\beta$ prescribes presentation of documents $\beta(a)$ in state $a$ and $\beta(b)$ in state $b$. It may be that $E$ is a $J$-deviation from $\beta(a)$ in state $a$, while $E$ is a $K$-deviation from $\beta(b)$ in state $b$. If there is a function $m$ with respect to which $\beta$ is ISC, then it must be that $m$ deters the $J$-coalition from deviating to $E$ in state $a$ and also deters the $K$-coalition from deviating to $E$ in state $b$. We use Lemma 1 to translate this constraint into an inequality defined for $E$ and $\beta$.

For any disclosure rule $\beta$, each $i \in N$, and $E \in \mathcal{D}$, let

$$
B(i ; \beta, E) \equiv\{a \in A \mid i \notin R(a ; \beta(a), E)\}
$$

This is the set of states at which player $i$ is not needed to induce disclosure of $E$ by deviation from the prescription of $\beta$. Note that $E \not \subset D(a)$ implies $a \notin B(i ; \beta, E)$. For any $a \in B(i ; \beta, E)$, using Lemma 1 , we know that player $i$ 's transfer when $E$ is disclosed must be at least as great as his transfer when $\beta(a)$ is disclosed; further, 
the latter transfer is supposed to be $g_{i}(a)$, given the transfer function $g$. Examining all states in $B(i ; \beta, E)$, we have the following lower bound on player $i$ 's transfer conditional on $E$.

$$
z_{i}(E ; \beta, g) \equiv\left\{\begin{array}{ll}
\max _{a \in B(i ; \beta, E)} g_{i}(a) & \text { if } B(i ; \beta, E) \neq \emptyset \\
-\infty & \text { if } B(i ; \beta, E)=\emptyset
\end{array} .\right.
$$

Here is our characterization of implementability for a given disclosure rule.

Lemma 2: Take as given a disclosure rule $\beta$ and a transfer function $g$. There exists an externally enforced contract $m$ such that $(i) \beta$ is an ISC disclosure rule with respect to $m$ and (ii) $g(a)=m(\beta(a))$ for every $a \in A$, if and only if

$$
\sum_{i \in N} z_{i}(E ; \beta, g) \leq 0, \text { for every } E \in \mathcal{D} .
$$

To prove the "sufficiency" direction of this lemma, suppose that (i) and (ii) hold for some $m$. Take any $E \in \mathcal{D}$. Using the definition of $z_{i}$, The ISC condition implies that $m_{i}(E) \geq z_{i}(E ; \beta, g)$ for every player $i$. By balancedness of the transfers, we have $\sum_{i \in N} m_{i}(E)=0$, which implies condition (1). To prove the "necessity" direction of Lemma 2, suppose condition (1) holds. For each $E \in \mathcal{D}$, we can find a vector $m(E) \in \mathbf{R}_{0}^{n}$ such that $m_{i}(E) \geq z_{i}(E ; \beta, g)$ for every player $i$. It is not difficult to confirm that $m(\beta(a))$ must equal $g(a)$, for each $a \in A$. By the definition of $z_{i}$ and Lemma 1, we thus have (i) and (ii).

We use Lemma 2 to prove Theorem $2^{\prime}$. Suppose transfer function $g$ is implemented by disclosure rule $\beta$. Using Lemma 2 , to ascertain whether $g$ is also implemented by $\bar{\beta}$, we need to check whether

$$
\sum_{i \in N} z_{i}\left(E^{\prime} ; \bar{\beta}, g\right) \leq 0, \text { for all } E^{\prime} \in \mathcal{D}
$$

To do this, consider any set of documents $E^{\prime} \in \mathcal{D}$. If $z_{i}\left(E^{\prime} ; \bar{\beta}, g\right)=-\infty$ for some $i$, then

$$
\sum_{i \in N} z_{i}\left(E^{\prime} ; \bar{\beta}, g\right) \leq 0
$$

is assured. Therefore, suppose $z_{i}\left(E^{\prime} ; \bar{\beta}, g\right) \neq-\infty$, for all $i \in N$. Then for each player $i$, let $a^{i}$ be a state such that $a^{i}$ maximizes $g_{i}(a)$ over all $a \in B\left(i, \bar{\beta}, E^{\prime}\right)$. Then we can define $E$ so that $E_{i}=\beta_{i}\left(a^{i}\right)$ for all $i$. This implies that player $i$ is not needed to deviate to $E$ from $\beta\left(a^{i}\right)$ at $a^{i}$, unless we have the non-feasibility case where $E \not \subset D\left(a^{i}\right)$. However, we can rule out $E \not \subset D\left(a^{i}\right)$ by the definition of $B$ and since $a^{i} \in B\left(i, \bar{\beta}, E^{\prime}\right)$. This is because (i) $E^{\prime} \subset D\left(a^{i}\right)$ for every $i \in N$ and (ii) we have $E_{i}=\beta_{i}\left(a^{i}\right) \subset D_{i}\left(a^{i}\right)=E_{i}^{\prime}$ for all $i$. Thus, $E_{i} \subset E_{i}^{\prime}$ for all $i \in N$, which implies that $E \subset D\left(a^{i}\right)$ for all $i \in N$. We conclude that $a^{i} \in B(i, \beta, E)$. Thus

$$
z_{i}(E ; \beta, g) \geq z_{i}\left(E^{\prime} ; \bar{\beta}, g\right) \text { for all } i \in N
$$


which implies that

$$
\sum_{i \in N} z_{i}\left(E^{\prime} ; \bar{\beta}, g\right) \leq \sum_{i \in N} z_{i}(E ; \beta, g) \leq 0,
$$

for all $E^{\prime} \in \mathcal{D}$. That $g$ is implemented by ISC disclosure implies (2). Q.E.D.

Note how Theorem $2^{\prime}$ simplifies checking whether a given transfer function can be implemented by an ISC disclosure rule. One need only verify the condition (1) for the full disclosure rule.

Theorem 4': Every implementable transfer function is measurable with respect to $P^{D}$. Furthermore, for any $i \in N$, every transfer function that is measurable with respect to $\bar{P}^{D_{i}}$ is implementable.

Proof: Theorem $2^{\prime}$ implies that measurability with respect to $P^{D}$ is necessary for implementability, proving the first claim. The second claim is proved using the same argument employed in the proof of Theorem 4, with " $i$ " in place of " $j . "$ Q.E.D.

Theorem 1": Assume the state and evidence environment is complete. A transfer function $g$ is implemented by an ISC disclosure rule if and only if, for every subset of players $J \subset N$ and every pair of states $a$ and $b, G_{J}(a)>G_{J}(b)$ implies that either a player in group $J$ can positively distinguish $a$ from $b$ or a player in group $-J$ can positively distinguish $b$ from $a$, or both.

Proof: We only need to prove the "necessity" direction, because the "sufficiency" direction is established by Theorem $1^{\prime}$. Take any transfer function $g$ that satisfied the condition on positive distinctions. For each $E \in \mathcal{D}$, simply define $m(E)=g(a)$ for that state $a$ for which $D(a)=E$. Then it is easy to check that $\bar{\beta}$ is an ISC disclosure rule with respect to $m$. Suppose that $E$ is a $J$-deviation from $\bar{\beta}(a)$ at state $a$. Let $b$ be such that $D(b)=E$. We have $D_{J}(b) \subset D_{J}(a)$ and $D_{-J}(a)=D_{-J}(b)$. Thus, no one in group $J$ can positively distinguish $b$ from $a$ and no one in group $-J$ can positively distinguish $a$ from $b$. Because $g$ satisfies the condition on positive distinctions, we have $G_{J}(a) \geq G(b)$, which means that the $J$-coalition has no incentive to deviate from $\bar{\beta}$. Q.E.D.

Theorem $3^{\prime \prime}$ : The following is true for any document set $\underline{D}$ that has the breadth property. A transfer function $g$ is implementable with ISC disclosure if and only if it can be implemented with ISC disclosure on $\underline{D}$. Furthermore, if a document set $\underline{D}^{\prime}$ does not have the breadth property, then there is a transfer function that is implementable with ISC disclosure but not on $\underline{D}^{\prime}$.

Proof: The arguments used to prove Theorem 3 readily extend to this version of the result. Q.E.D. 


\section{B Productive Interaction}

In the body of this paper, our analysis is geared toward understanding behavior in periods 3 and 4 of the contractual relationship. In this appendix, we turn our attention to interaction in periods 1 and 2 and we take a broader perspective on the components of contract. Let us presume a simple specification of productive interaction. Suppose that in period 2 the players simultaneously and independently select actions. Player $i$ 's action space is denoted $A_{i}$; we define $A \equiv A_{1} \times A_{2} \times \cdots \times A_{n}$. In other words, in period 2 the agents play a one shot "production" game with action profiles $A$ and payoffs given by $u$ plus the continuation value from period 3 . The resulting action profile $a=\left(a_{1}, a_{2}, \ldots, a_{n}\right)$ represents the state of the relationship at the end of period 2.

In period 1, before playing the production game, the players jointly agree to a contract. The contract has two components: an externally enforced part $m$ and a self-enforced part which describes the disclosure rule $\beta$ and behavior in the production phase. We suppose that $\beta$ is an ISC disclosure rule with respect to $m$. Thus, interaction in periods 3 and 4 can be summarized by the implied transfer function $g$. In period 1, the players indirectly select a transfer function $g$ through their selection of $m$ and their coordination on $\beta$. This justifies viewing interaction in period 2 as a game with action profiles $A$ and payoffs given by $u+g$, where $g$ is any implementable transfer function. We analyze this contracting game by determining whether there is an implementable transfer function that facilitates self-enforcement of a given $a^{*}$-in other words, that makes $a^{*}$ a Nash equilibrium of period 2 interaction. ${ }^{19}$

Given $a^{*} \in A$, let $F\left(a^{*}\right)$ be the set of (balanced) transfer functions that induce $a^{*}$ as a Nash equilibrium of the production phase. Mathematically, $g \in F\left(a^{*}\right)$ if and only if $g$ is balanced and

$$
u_{i}\left(a^{*}\right)+g_{i}\left(a^{*}\right) \geq u_{i}\left(a_{i}, a_{-i}^{*}\right)+g_{i}\left(a_{i}, a_{-i}^{*}\right),
$$

for each player $i$ and each $a_{i} \in A_{i}$. Let $F^{D}$ be the set of implementable transfer functions. Clearly, there is a contract that yields action profile $a^{*}$ if and only if $F\left(a^{*}\right) \cap F^{D} \neq \emptyset$. Also note

Lemma 3: If $g \in F^{D}$ and, for some balanced vector $\phi \in \mathbf{R}^{n}, g^{\prime}$ is defined by $g^{\prime}(a)=$ $g(a)+\phi$ for each a, then $g^{\prime} \in F^{D}$ as well.

In words, adding a constant transfer between the players does not disrupt implementability, since incentives in period 3 are not altered. Thus, in period 1, the players implement a transfer function associated with the solution of

$$
\max _{a^{\prime} \in A^{*}} \sum_{i \in N} u_{i}\left(a^{\prime}\right), \text { where } A^{*} \equiv\left\{a^{*} \in A \mid F\left(a^{*}\right) \cap F^{D} \neq \emptyset\right\} .
$$

\footnotetext{
${ }^{19}$ Here we focus on enforcing pure action profiles. The Appendix of our 2001 working paper contains analysis of mixed action profiles and moves of nature. One can easily extend the analysis to more complicated period 2 interaction, using the appropriate equilibrium concept. For example, production in period 2 may be modeled as a dynamic game. We simply require that all productive activity takes place in period 2, prior to evidence disclosure.
} 
This maximization problem yields the highest joint value that can be attained in the contractual relationship, given the incentive constraints in the production and evidence disclosure phases. The joint value can be arbitrarily allocated between the players, given Lemma 3.

As explored earlier, sometimes it is helpful to analyze the set of transfer functions that are measurable with respect to a particular partition of the state space. Given a partition $P$, we can provide a necessary and sufficient condition for $a^{*}$ to be induced by a transfer function that is measurable with respect to $P$. The condition relates to the function

$$
w_{i}\left(a, a^{*}\right) \equiv\left\{\begin{array}{ll}
-\infty & \text { if } Q^{i}\left(a^{*}\right) \cap P(a)=\emptyset \\
\max \left\{u_{i}\left(a_{i}^{\prime}, a_{-i}^{*}\right) \mid\left(a_{i}^{\prime}, a_{-i}^{*}\right) \in P(a)\right\}-u_{i}\left(a^{*}\right) & \text { if } Q^{i}\left(a^{*}\right) \cap P(a) \neq \emptyset
\end{array},\right.
$$

where $Q^{i}\left(a^{*}\right) \equiv \cup_{a_{i} \in A_{i}} P\left(a_{i}, a_{-i}^{*}\right)$. The function $w_{i}$ represents the maximal increase in productive payoff that can be achieved by player $i$ by unilaterally deviating from $a^{*}$ in a way that yields an action profile in the partition element $P(a)$. Let $\hat{F}(P)$ be the set of transfer functions that are measurable with respect to $P$.

Theorem 6: Consider any partition $P$ of the action space. Then $F\left(a^{*}\right) \cap \hat{F}(P) \neq \emptyset$ if and only if

$$
\sum_{i \in N} w_{i}\left(a, a^{*}\right) \leq 0, \text { for all } a \in A \text {. }
$$

Theorem 6 is closely related to Theorem 1 of Legros and Matthews (1993). ${ }^{20}$ The intuition for necessity is simple. As transfers must be balanced due to renegotiation, summing each player's Nash equilibrium condition gives the result. In the sufficiency direction, we consider separately each element of the partition of $A$. If players generally do better by deviating to the element $P(a)$ than by being in $P\left(a^{*}\right)$ (i.e., $\left.\sum_{i \in N} w_{i}\left(a, a^{*}\right)>0\right)$, then we cannot expect to induce play of $a^{*}$ as a Nash equilibrium with any transfer function $g$ as we require $\sum_{i \in N} g_{i}=0$. That is, there does not exist a transfer function $g$ that sufficiently punishes all players for unilateral deviation. However, when $\sum_{i \in N} w_{i}\left(a, a^{*}\right) \leq 0$ there does exist a transfer function that is measurable with respect to $P$ and prevents deviation to the element of the partition $P(a)$.

\section{References}

Bentham, J. (1827), Rationale of Judicial Evidence, Hunt and Clarke: London. Bernardo, A., E. Talley, and I. Welch (2000), "A Theory of Legal Presumptions," Journal of Law, Economics, and Organization 16: 1-49.

\footnotetext{
${ }^{20}$ By specifying that an individual player appropriates the verifiable partnership output and confining attention to finite actions, Legros and Matthew's result is seen as a special case of ours.
} 
Bernheim, B. D., B. Peleg, and M. Whinston (1987), "Coalition-Proof Nash Equilibria I. Concepts," Journal of Economic Theory, 42: 1-12.

Bernheim, B. D. and M. Whinston (1999), "Incomplete Contracts and Strategic Ambiguity," American Economic Review, 88: 902-932.

Brazil, W. (1978), "The Adversary Character of Civil Discovery: a Critique and Proposals for Change," Vanderbilt Law Review 31: 1295.

Brennan, J. and J. Watson (2002), "The Renegotiation-Proofness Principle and Costly Renegotiation," UC San Diego Economics Working Paper 2002-10.

Bull, J. (2001), "Costly Evidence Production and the Limits of Verifiability," manuscript, The Wharton School, University of Pennsylvania.

Bull, J. and J. Watson (2001), "Evidence Disclosure and Verifiability," UC San Diego Working Paper 2000-16R, revised 2001.

Bull, J. and J. Watson (2002), "Hard Evidence and Mechanism Design," UC San Diego Working Paper.

California Evidence Code.

Cooter, R. D. and D. L. Rubinfeld (1989), "Economic Analysis of Legal Disputes," Journal of Economic Literature 23: 1067-97.

Cooter, R. D. and D. L. Rubinfeld (1994), "An Economic Model of Legal Discovery," Journal of Legal Studies 23: 435-463.

Cooter, R. D. and D. L. Rubinfeld (1995), "Reforming The New Discovery Rules," The Georgetown Law Journal 84: 61-89.

Daughety, A. and J. Reinganum (2000a), "Appealing Judgments," RAND Journal of Economics 31: 502-525.

Daughety, A. and J. Reinganum (2000b), "On the Economics of Trials: Adversarial Process, Evidence, and Equilibrium Bias," Journal of Law, Economics, and Organization 16: 365-394.

Federal Rules of Evidence.

Froeb, L. and B. Kobayashi (2000), "Evidence Production in Adversarial vs. Inquisitorial Regimes," Vanderbilt University Law School, Working Paper 99-13.

Green, J. and J. Laffont (1986), "Partially Verifiable Information and Mechanism Design," Review of Economic Studies 53: 447-456.

Hart, O. and J. Moore (1988), "Incomplete Contracts and Renegotiation," Econometrica 56: $755-785$.

Holmström, B. (1982), "Moral Hazard in Teams," Bell Journal of Economics 13: 324-340.

Legros, P. and S. Matthews (1993), "Efficient and Nearly-Efficient Partnerships," Review of Economic Studies 68: 599-611.

Lipman, B. L. and D. J. Seppi (1995), "Robust Inference in Communication Games with Partial Provability," Journal of Economic Theory 66: 370-405. 
Maskin, E., "Nash Equilibrium and Welfare Optimality," Review of Economic Studies 66 (1999): 23-38.

Maskin, E. and Moore (1999), "Implementation and Renegotiation," Review of Economic Studies 66: 39-56.

Milgrom, P. and J. Roberts (1986), "Relying on the Information of Interested Parties," RAND Journal of Economics 17: 18-32.

Okuno-Fujiwara, M., A. Postlewaite, and K. Suzumura (1990), "Strategic Information Revelation," Review of Economic Studies 57: 25-47.

Posner, R. (1999), "An Economic Approach to the Law of Evidence," Stanford Law Review 51: 1477-1546.

Rubinfeld, D. and D. Sappington (1987), "Efficient Awards and Standards of Proof in Judicial Proceedings," RAND Journal of Economics, 18: 308-315.

Saltzburg, S. (1978), "A Special Aspect of Relevance: Countering the Negative Inference Associated with the Absence of Evidence," California Law Review 66: 1011-1060.

Sanchirico, C. (1999) "Games, Information, and Evidence Production with Application to English Legal History," manuscript, University of Virginia.

Seidmann, D.J. and E. Winter (1997), "Strategic Information Transmission with Verifiable Messages," Econometrica 65: 163-169

Schwartz, A. and J. Watson (2001), "The Law and Economics of Costly Contracting" UC San Diego Working Paper 2001-21.

Shapiro, D. (1979), "Some Problems of Discovery in an Adversary System," Minnesota Law Review 63: 1055-1100.

Shin, H. S. (1998), "Adversarial and Inquisitorial Procedures in Arbitration," Rand Journal of Economics 29: 378-405.

Shin, H. S. (1994), "The Burden of Proof in a Game of Persuasion," Journal of Economic Theory 64: 253-264.

Sobel, J. (1985), "Disclosure of Evidence and Resolution of Disputes," in A.E. Roth Game Theoretic Models of Bargaining, Cambridge: Cambridge University Press.

State v. Simons, 17 N. H. 83, 88 (1845).

Teply, L. and R. Whitten (2000), Civil Procedure 2nd. ed. New York: Foundation Press.

Tullock, G. (1980), Trials on Trial. New York: Columbia University Press.

Watson, J. (2001), "Contract, Mechanism Design, and Technological Detail," UC San Diego Economics Working Paper 2002-04.

Wigmore, J. (1935), A Student's Textbook on the Law of Evidence, Brooklyn: The Foundation Press, Inc.

Wigmore, J. (1940), Evidence in Trials at Common Law, Third Ed. Boston: Little, Brown. 